\title{
Expression of Relation of Medical Institutions and the Simulation of the Relation Recovery for Disaster
}

\author{
S. Mizuno \\ Shizuoka Institute of Science and Technology
}

\author{
M. Seki \\ Shizuoka University
}

\author{
Y. Fujisawa \\ University of Shizuoka \\ A. Kakehi \\ Kogakuin University
}

\begin{abstract}
The present medical institution cooperate each other, such as specialty skills or regional peculiarity. However, when disasters, such as an earthquake, an institution may not have function as a medical institution and the balance of the medical service at the time will usually unstable. Restoring medical service, each medical institution also becomes to be stable. In this research, we express relation of the medical institutions as a medical network. Moreover, we get the simulation of the degree of relation of a medical institution. By expressing a medical network, it becomes clear where it needs medical services. And it becomes possible where we should focus to advance restoration by medical network simulation.
\end{abstract}

Keywords-queue; simulation

\section{INTRODUCTION}

Currently, medical institutions collaborate with various specialties and areas to provide a range of medical services. However, some medical facilities have lost the ability to function properly following a disaster such as an earthquake; thus, the balance of normal medical services has collapsed. As recovery progresses, each medical institution restore its ability to provide services and return to normalcy. This research aims to describe the connection among medical institutions in normal conditions as a functional medical network and simulate the degree of their connections when restoring medical services following a disaster. This simulation makes possible the clarification of them edictal services that are insufficient in the medical network and the degree of connection during a disaster to facilitate the prioritization of restoration efforts.

\section{MODELING BY A CLOSED QUEUING NETWORK}

In this study, we modeled a closed queuing network using hospital capacities and geographic locations, and we used the system to determine the frequency of hospital use by customers. With this model, the closed queuing network [1] [2] focused solely on the movement within the system to determine the overall hospital system equilibrium within a local area. The study considered:

i. The type of customer using the network services in the network,

\section{ii. $K$ sites within the network, and}

iii.that the system would have a finite number of total customers $(N)$, to satisfy the equation given below, as the number of customers at site $k$ :

$$
N=\sum_{k=1}^{K} n_{k}
$$

Where $n_{k}$ is the number of customers at site $k$.

iv.At site $k$, the service time followed an exponential distribution of service rate $\mu_{k}$.

$v$.The rate of completed arrivals of customers from the inner network at site $k$ is $\alpha_{k}$.

vi.Customers who received service at site $i$ move to site $j$ with the probability as follows:

$$
p_{i j} \quad\left(1 \leq i, j \leq K, p_{i j} \geq 0, \sum_{j=1}^{K} p_{i j}=1\right)
$$

We defined the service rate at each site as users' utilization frequency. In addition, $\alpha_{k}$ must satisfy the equation as follows:

$$
\begin{aligned}
& x_{1}=1, x_{k}=\sum_{j=1}^{k} x_{j} p_{j k},(k=2,3, \ldots, K) \\
& \text { Moreover, } \rho_{k}=\alpha_{k} / \mu_{k},(\mathrm{k}=1,2, \ldots, \mathrm{K})
\end{aligned}
$$

Therefore, the stationary distribution in which there are $\mathrm{n} 1$ people in facility $1, \ldots, n_{k}$ people in facility $\mathrm{k}$ is as follows:

$$
\begin{aligned}
& \quad \pi\left(n_{1}, n_{2}, \ldots, n_{k}\right)=1 / G(N, K) \prod_{k=1}^{K} \rho_{k}^{n_{k}} \\
& \text { Where } G(N, K)=\sum_{n_{1}+. . n_{k}=N} \prod_{k=1}^{K} \rho_{k}^{n_{k}}
\end{aligned}
$$

We calculated transition probability $p_{i j}$ according to the gravity model as follows:

$f_{i j}$ : The amount of movement from region $\mathrm{i}$ to region $\mathrm{j}$

$p_{i}$ : The total amount of movement from region $i$ as the departure place

$q_{j}$ : The total amount of movement from region $\mathrm{j}$ as the arrival place

$d_{i j}$ : The distance between regions $\mathrm{i}$ and $\mathrm{j}$

C: The gravity model normalization constant: 0.145722117443322 


$$
f_{i j}=C \frac{p_{i}^{a} q_{j}^{b}}{d_{i j}^{c}} \quad, \quad i \in K, j \in K
$$
$\mathrm{c}=0.5$.

In this study, we performed calculations with $\mathrm{a}=\mathrm{b}=1$ and
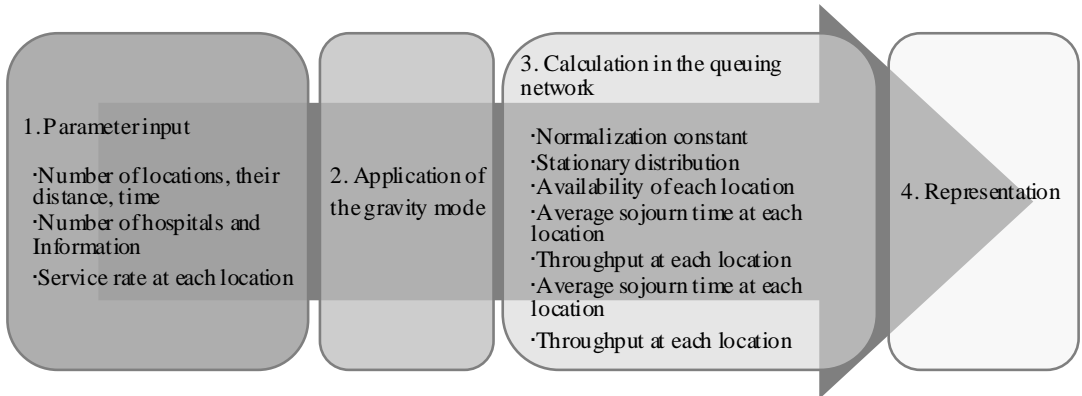

FIGURE I. SYSTEM FLOW

\section{EXAMPLE OF NUMERICAL CALCULATIONS}

The numerical calculations for this study were performed as follows:

- Four disaster base hospitals and eight emergency response hospitals in Hamamatsu City were used, as shown in Table 1.

- The population distribution used in the gravity model define swards as units, as shown in Table2

- When multiple sites within the same ward were used, we divided the population by the number of used sites within

\section{SYSTEM FLOW}

The proposed system flow for this study is shown in Figure 1 First, we selected sites for inclusion in this system. Then, based on population information and the distance between sites, we determined the transition probability using the gravity model. Subsequently, we calculated the average wait number, which is considered to be the time required for the hospital, at each site in the queuing network.

TABLE I. FOUR DISASTER BASE HOSPITALS AND EIGHT EMERGENCY RESPONSE HOSPITALS.

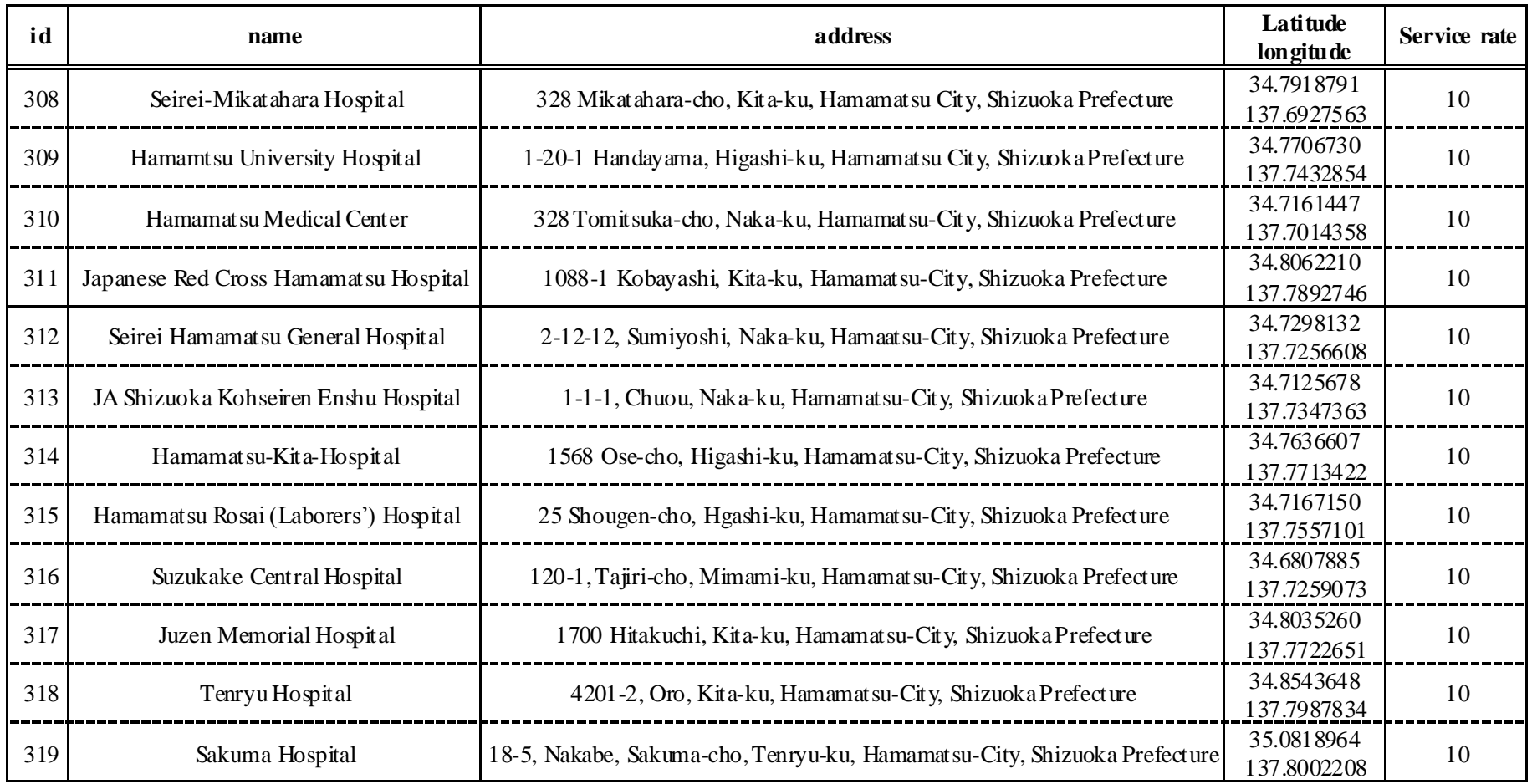


TABLE II. POPULATION IN HAMAMATSU CITY FOR EACH WARD

\begin{tabular}{|l|l|}
\hline Ward & Population \\
\hline \hline Naka-ku, Hamamatsu City & 232,479 persons \\
\hline Kita-ku, Hamamatsu City & 93,799 persons \\
\hline Minami-ku, Hamat su City & 98,428 persons \\
\hline Tenryu-ku, Hamamat su City & 33,490 persons \\
\hline Higashi-ku, Hamamat su City & 125,745 persons \\
\hline Kita-ku, Hamamatsu City & 92,691 persons \\
\hline Nishi-ku, Hamamatsu City & 107,677 persons \\
\hline
\end{tabular}

A. A Case where Four Disaster Base Hospitals and Eight

\section{Emergency Response Hospitals are Used}

We considered a case in which four disaster base hospitals and eight emergency response hospitals were used in normal non-disaster conditions. Where there are several hospitals within the same ward, the allocated population is equal to the ward population divided by the nu mber of hospitals within the ward. Table 3 shows a transition probability matrix obtained from the gravity model using the population assigned and the transport distance or the travel time between hospitals. The transport distance and the travel time between hospitals were automatically determined using Google Map API. The order of elements in the matrix corresponds to their IDs shown in Table 1. In addition, we calculated the transition probability matrix shown in Table 3 on the basis of the transport distance.

TABLE III. TRANSITION PROBABILITY MATRIX OBTAINED FROM THE GRAVITY MODEL.

\begin{tabular}{|c||c|c|c|c|c|c|c|c|c|c|c|c|}
\hline 308 & 308 & 309 & 314 & 315 & 310 & 312 & 313 & 311 & 317 & 318 & 316 & 319 \\
\hline \hline 308 & 0 & 0.2083 & 0.0878 & 0.0571 & 0.0908 & 0.207 & 0.1118 & 0.0465 & 0.0752 & 0.0284 & 0.0843 & 0.0027 \\
\hline 309 & 0.1676 & 0 & 0.3508 & 0.0484 & 0.0606 & 0.1294 & 0.0934 & 0.0277 & 0.0526 & 0.0126 & 0.0561 & 0.0009 \\
\hline 314 & 0.0746 & 0.3704 & 0 & 0.0785 & 0.0529 & 0.1117 & 0.1 & 0.0648 & 0.101 & 0.0185 & 0.0267 & 0.0009 \\
\hline 315 & 0.0247 & 0.026 & 0.0399 & 0 & 0.0939 & 0.2361 & 0.461 & 0.0091 & 0.0103 & 0.0028 & 0.0959 & 0.0004 \\
\hline 310 & 0.03 & 0.0249 & 0.0206 & 0.0718 & 0 & 0.3248 & 0.3828 & 0.006 & 0.0082 & 0.0038 & 0.1269 & 0.0004 \\
\hline 312 & 0.048 & 0.0372 & 0.0305 & 0.1268 & 0.2279 & 0 & 0.4117 & 0.0059 & 0.0098 & 0.0037 & 0.0982 & 0.0003 \\
\hline 313 & 0.0211 & 0.0219 & 0.0222 & 0.2016 & 0.2186 & 0.3351 & 0 & 0.0043 & 0.0062 & 0.0026 & 0.1661 & 0.0003 \\
\hline 311 & 0.0672 & 0.0497 & 0.1101 & 0.0304 & 0.0263 & 0.0368 & 0.0332 & 0 & 0.5482 & 0.0754 & 0.021 & 0.0016 \\
\hline 317 & 0.0909 & 0.079 & 0.1437 & 0.0288 & 0.0302 & 0.0509 & 0.0398 & 0.4589 & 0 & 0.0457 & 0.0308 & 0.0012 \\
\hline 318 & 0.1332 & 0.0732 & 0.1021 & 0.0309 & 0.0538 & 0.0755 & 0.0645 & 0.2445 & 0.1769 & 0 & 0.039 & 0.0065 \\
\hline 316 & 0.0393 & 0.0325 & 0.0146 & 0.1036 & 0.179 & 0.1975 & 0.4103 & 0.0068 & 0.0119 & 0.0039 & 0 & 0.0007 \\
\hline 319 & 0.1874 & 0.0763 & 0.0721 & 0.0593 & 0.0749 & 0.0768 & 0.0995 & 0.0752 & 0.0717 & 0.0969 & 0.11 & 0 \\
\hline
\end{tabular}

Table 4represents the degree of relationship among the hospitals when all 12 sites are used. We calculated the result using the distance between the population distribution and the hospital. Although there were many factors that could be considered, including the number of doctors and nurses and their skill levels at these hospitals, we considered that the distance and population distribution were the greater factors when anticipating a disaster. In this case, the value of Enshu Hospital is very high, indicating that this hospital is located in a geographically and demographically key location.

\section{B. A Case in which only Four Disaster Base Hospitals Sites are in Operation}

Next, we considered a case in which emergency response hospitals are not available and only disaster base hospitals are operating.
In this case, the value of the Hamamatsu University Hospital located in the centre of the map is greater than the others, as shown in table 4 . The degree of relationship among the disaster hospitals in this case is also greater than that in the 12 sites case.

\section{Degree of Relationship among Hospitals when Emergency Response Hospitals become Available}

We next considered the degree of relationship among hospitals when emergency response hospitals become sequentially available. For this case, we performed the calculations with the assumption that hospitals with lower values shown in Table 4 would become available earlier than those with higher values. The case in which 12 sites were used is assumed to be the normal situation. It shows hospitals serving as relationship centres when sites are added one-byone, assuming that the number of sites is four for a condition under which only disaster base hospitals are available during 
an earthquake. In this case, we found that central sites interchange through the influence of the locations of hospitals and populations as hospitals are progressively added.

Table 4 show the changes in the degrees of relationship due to the recovery of sites. This result indicates that the degree of relationship is also changed by the added hospitals when medical institutions are in the process of recovery. We consider these data to be essential information for establishing a chain of command following a disaster.

TABLE IV. CHANGE IN THE DEGREES OF RELATIONSHIP DUE TO THE RECOVERY OF SITES.

\begin{tabular}{|c||c|c|c|c|c|c|c|c|c|}
\hline Name of base & $\mathbf{1 2}$ & $\mathbf{4}$ & $\mathbf{5}$ & $\mathbf{6}$ & $\mathbf{7}$ & $\mathbf{8}$ & $\mathbf{9}$ & $\mathbf{1 0}$ & $\mathbf{1 1}$ \\
\hline \hline Seirei-Mikat ahara Hospital & 0.232 & 1.802 & 1.806 & 1.952 & 1.744 & 0.927 & 0.758 & 0.481 & 0.296 \\
\hline Hamamtsu University Hospital & 0.306 & 95.349 & 95.329 & 94.980 & 94.531 & 77.086 & 6.372 & 0.724 & 0.377 \\
\hline Hamamat su Medical Center & 1.332 & 2.235 & 2.240 & 2.446 & 2.089 & 1.414 & 85.790 & 94.989 & 3.529 \\
\hline Japanese Red Cross Hamamatsu Hospital & 0.150 & 0.614 & 0.617 & 0.385 & 0.628 & 0.632 & 0.477 & 0.344 & 0.179 \\
\hline Seirei Hamamatsu General Hospital & 4.375 & 0.000 & 0.000 & 0.000 & 0.000 & 0.000 & 0.000 & 0.000 & 93.862 \\
\hline JA Shizuoka Kohseiren Enshu Hospital & 91.632 & 0.000 & 0.000 & 0.000 & 0.000 & 0.000 & 0.000 & 0.000 & 0.000 \\
\hline Hamamatsu-Kita-Hospital & 0.285 & 0.000 & 0.000 & 0.000 & 0.000 & 18.924 & 3.975 & 0.655 & 0.341 \\
\hline Hamamatsu Rosai Hospital & 0.777 & 0.000 & 0.000 & 0.000 & 0.000 & 0.000 & 0.000 & 0.748 & 0.560 \\
\hline Suzukake Central Hospital & 0.680 & 0.000 & 0.000 & 0.000 & 0.000 & 0.000 & 1.862 & 1.537 & 0.581 \\
\hline Juzen Memorial Hospital & 0.185 & 0.000 & 0.000 & 0.000 & 0.862 & 0.878 & 0.649 & 0.432 & 0.223 \\
\hline Tenryu Hospital & 0.042 & 0.000 & 0.000 & 0.228 & 0.139 & 0.132 & 0.110 & 0.084 & 0.049 \\
\hline Sakuma Hospital & 0.003 & 0.000 & 0.008 & 0.008 & 0.008 & 0.007 & 0.007 & 0.005 & 0.003 \\
\hline
\end{tabular}

\section{The Model that Added Hospital Information}

We consider the model that added hospital information. In this case, the information added is the number of beds and the number of medical departments of the hospital. We define following variables.

$$
\begin{aligned}
& T_{i} \text { : The number of beds of the hospital i. } \\
& S_{i} \text { : The number of med ical departments of the hospital } i .
\end{aligned}
$$

Thus, we modify the gravity model equation.

$$
\begin{gathered}
f_{i j}=C \frac{\left(p_{i}+T_{i} \times S_{i} \times w\right)^{a} \times\left(q_{j}+T_{j} \times S_{j} \times w\right)^{b}}{d_{i j}^{c}} \\
i \in K, j \in K
\end{gathered}
$$

Where means that the weight variable of hospital information. We assume that number of medical examinees $T_{i} \times S_{i} \times w$. We use this modified model that included hospital information, we can get more precise information.

\section{CONCLUSION}

In this study, we expressed the balance among hospitals as a stream of customers using a closed queuing network, and based on their own assessments and geographical locations, we expressed the degrees of relationship among hospitals. However, hospital values must be evaluated from various perspectives such as the number of doctors and nurses, number of beds, and quality of their medical services. In a future study, we will achieve higher quality simulation by establishing additional ele ments for evaluation fro $m$ multiple perspectives.

\section{ACKNOWLEDGEMENT}

Special thanks go to Miss. Kanae Ohtsuka and Miss. Kyoko Kawamoto whose comments made enormous contribution to my work. Finally, This work was a part of results that supported by JSPS KAKENHI Grant Number 24560759 (Principle Investigator: Kakehi Atsuo).

\section{REFERENCE}

[1] Shinya Mizuno, Shogo Iwamoto, Naokazu Yamaki: Proposal of an Effective Computation Environment for the Traveling Salesman Problem Using Cloud Computing, Journal of Advanced Mechanical Design, Systems, and Manufacturing Vol. 6(2012) No. 5, pp.703-714.

[2] Shoji Kasahara: Queuing Theory and its Simulation. "Knowledge Base," Institute of Electronics, Information and Communication Engineers (2010).

[3] Shizuoka Prefecture: Fujinokuni Open Data Catalog, http://opendata.pref.shizuoka.jp/.

[4] Shinya Mizuno, Megumi Ishigami, Yui Maruyama, Yasuyuki Muramatsu, and Naokazu Yamaki: A proposed basic design for the opt imal placement of an electric two-wheeled vehicle using the queuing network, Scheduling Symposium. (2012): Collection of Lecture Articles, pp.9194. 